\begin{tabular}{|c|c|c|c|c|}
\hline 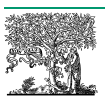 & Review & TRENDS in Plant Science & Vol.9 No.6 June 2004 & $\begin{array}{l}\text { Full text provided by www.sciencedirect.com } \\
\text { science } d \text { DiREct. }\end{array}$ \\
\hline
\end{tabular}

\title{
Signalling for developmental plasticity
}

\author{
Jorge J. Casal ${ }^{1}$, Christian Fankhauser ${ }^{2}$, George Coupland ${ }^{3}$ and Miguel A. Blázquez ${ }^{4}$ \\ ${ }^{1}$ IFEVA, Faculty of Agronomy, University of Buenos Aires, Av. San Martín 4453, 1417-Buenos Aires, Argentina \\ ${ }^{2}$ Département de Biologie Moléculaire, Sciences III, 30 quai E. Ansermet, 1211 Genève 4, Switzerland \\ ${ }^{3}$ Max Planck Institute for Plant Breeding, Carl von Linne Weg, 10, D-50829 Cologne, Germany \\ ${ }^{4}$ IBMCP, CSIC-Universidad Politécnica de Valencia, Av. de los Naranjos s/n, 46022 Valencia, Spain
}

\begin{abstract}
We describe some of the regulatory mechanisms found in the signalling networks that mediate plant plasticity in response to the environment. In the absence of the relevant environmental cue, the response is often actively repressed at multiple levels, including chromatin structure, mRNA stability and protein degradation. The environmental signal normally initiates several pathways that share signalling elements. Positive and/or negative regulatory loops are also activated. Such complex networks might provide robustness and buffer the system against environmental noise. Uncovering the functional significance of these networks is a timely challenge.
\end{abstract}

The range of environmental conditions that a higher animal has to face throughout its life is reduced by behavioural responses, including migration towards a more favourable location. By contrast, a plant cannot leave its place of origin and can be forced to face a wider range of conditions. Whereas the mature embryo of a higher animal contains many of the features of the adult individual, the result of plant embryogenesis is a seedling lacking most speciesspecific characteristics of the adult [1]. Spreading developmental decisions throughout the life cycle provides the opportunity to tune plant body form and function to the changing environment. A crucial feature of this strategy is the ability to anticipate the forthcoming conditions and initiate the developmental responses accordingly, often because of the perception of subtle environmental cues (Figure 1). Plants must combine the sensitivity required to respond to these signals with the ability to remain unaltered by environmental fluctuations that do not represent a signal. Here we review recent advances in our understanding of the signalling mechanisms that serve this purpose.

\section{Canalization in plants}

Canalization is the capacity to buffer normal development against deleterious alterations caused by mutations or fluctuations of the environment. Like other organisms, plants have sophisticated mechanisms of canalization, and therefore plasticity is not simply phenotypic variation caused by the inability to achieve canalization. The heatshock protein 90 (HSP90, GenBank Accession number Y07613) chaperones metastable 'client' proteins so that they are stable in conformations required for their function (e.g. signal transduction). Pharmacological inhibition of

Corresponding author: George Coupland (coupland@mpiz-koeln.mpg.de).
HSP90 uncovers different morphological phenotypes dependent on the underlying genetic variation in Arabidopsis seedlings. These phenotypes include differences in shape, colour and size of aerial organs, altered root morphology and shoot or root orientation [2]. HSP90 seems to act as a

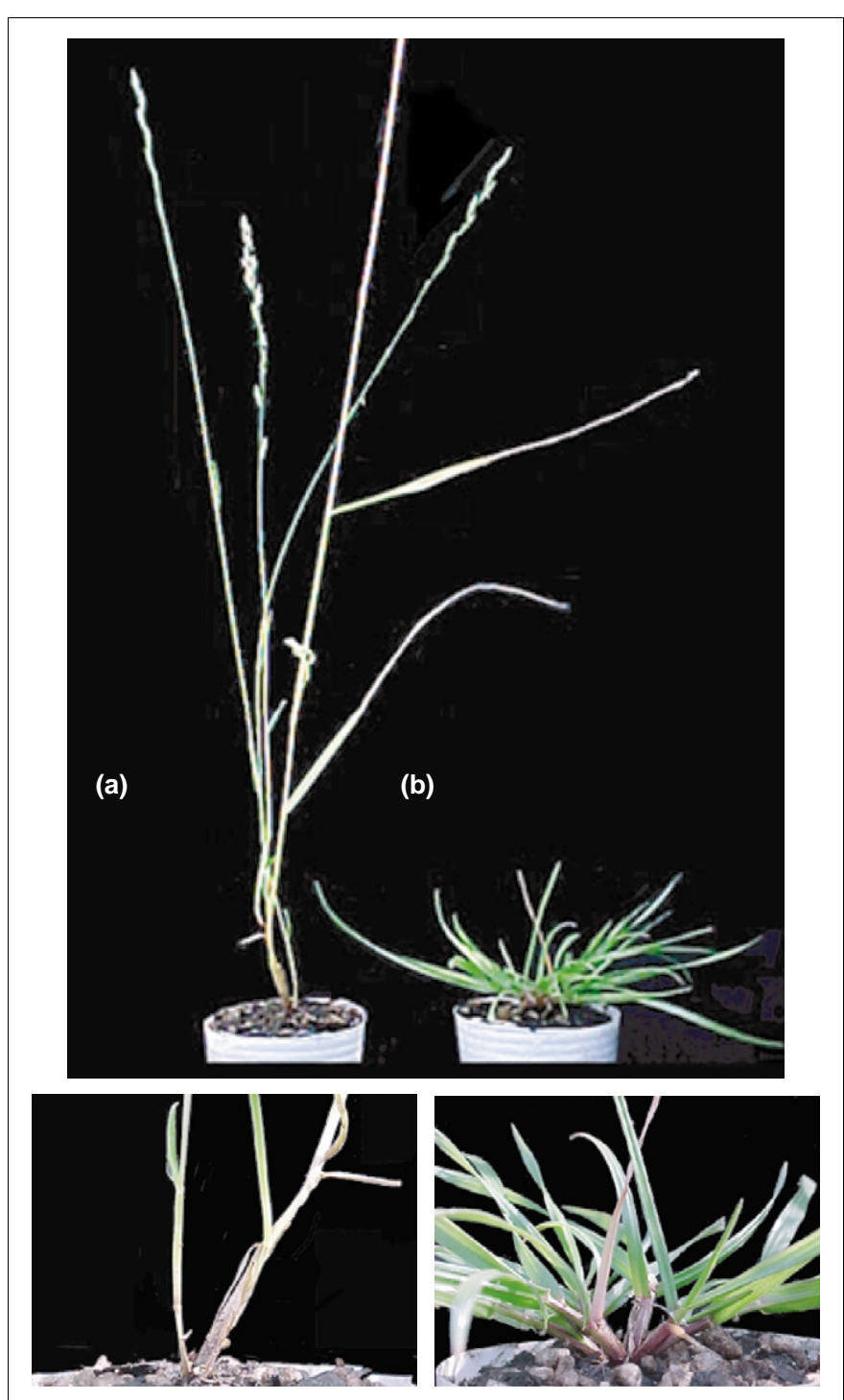

Figure 1. Plant plasticity. Plants of Lolium multiflorum were grown under sunlight (a) with or (b) without supplementary far-red light simulating the presence of neighbouring vegetation. Note differences in developmental stage (flower stalk under supplementary far-red (a), tillering and pigmentation. Details are shown in lower panels. Photographs courtesy of Hernán Ghiglione and Karina Oliverio (University of Buenos Aires, Argentina). 
buffer for developmental stability against genetic or environmental variation in Arabidopsis. This function appears to be important in evolution because under extreme environments the buffering capacity of HSP90 would be compromised releasing polymorphisms that could eventually provide variation appropriate to the environmental challenge. Based on this characteristic, HSP90 can be defined as an evolutionary capacitor.

Although HSP90 appears to be particularly well suited to suppress phenotypic variation, additional genes can complement this function. Canalization is an emergent property of complex developmental-genetic networks. Simulations validated with yeast experimental data demonstrate that a single gene knockout disrupting the network can exhibit a larger phenotypic variation than can the wild type [3]. The complexity of the genetic networks controlling plant development is well established and one of its sources is the occurrence of multigene families that, as observed for instance in the case of MADS-box genes in the control of ovule and carpel development, show partially redundant and divergent functions [4].

Whether a particular change in the environment does not influence plant development owing to canalization or is detected as a signal generating a response depends on the sensory and transductional mechanisms of the plant. The following sections describe key architectural features of these mechanisms.

\section{Multiplicity of pathways \\ Multiple environmental cues control flowering via partially divergent pathways}

Some developmental decisions, such as flowering, are based on the coordination of different environmental signals (Figure 2), which include day length and extended exposure to low winter temperatures. In the unfavourable conditions imposed by crowding, these coordinated cues can be overruled allowing rapid flowering in response to low red to far-red ratios (Figure 1).

Any fluctuation of the environment that correlates with circumstances that require adjustment of growth and development is potentially a signal. However, the fluctuation becomes a signal only if it is linked to the molecular events controlling the relevant developmental processes. Some Arabidopsis accessions accelerate flowering in response to exposure of the imbibed seeds or young seedlings to several weeks of low temperatures (vernalization), whereas others are unaffected by this signal. Winterannual accessions are late flowering because the FRIGIDA (FRI, Accession number AF228499) locus promotes the expression of the MADS box transcription factor FLOWERING LOCUS C (FLC, Accession number AF537203), a negative regulator of flowering, and vernalization accelerates flowering by down-regulating the expression of FLC. Null alleles of FRI [5] and natural allelic variation at the $F L C$ locus [6] have allowed the evolution of summer-annual accessions that flower early even in the absence of vernalization. Several of these FRI and FLC alleles have appeared independently during the course of evolution. The MADS AFFECTING FLOWERING 2 (MAF2, Accession number AY231441)

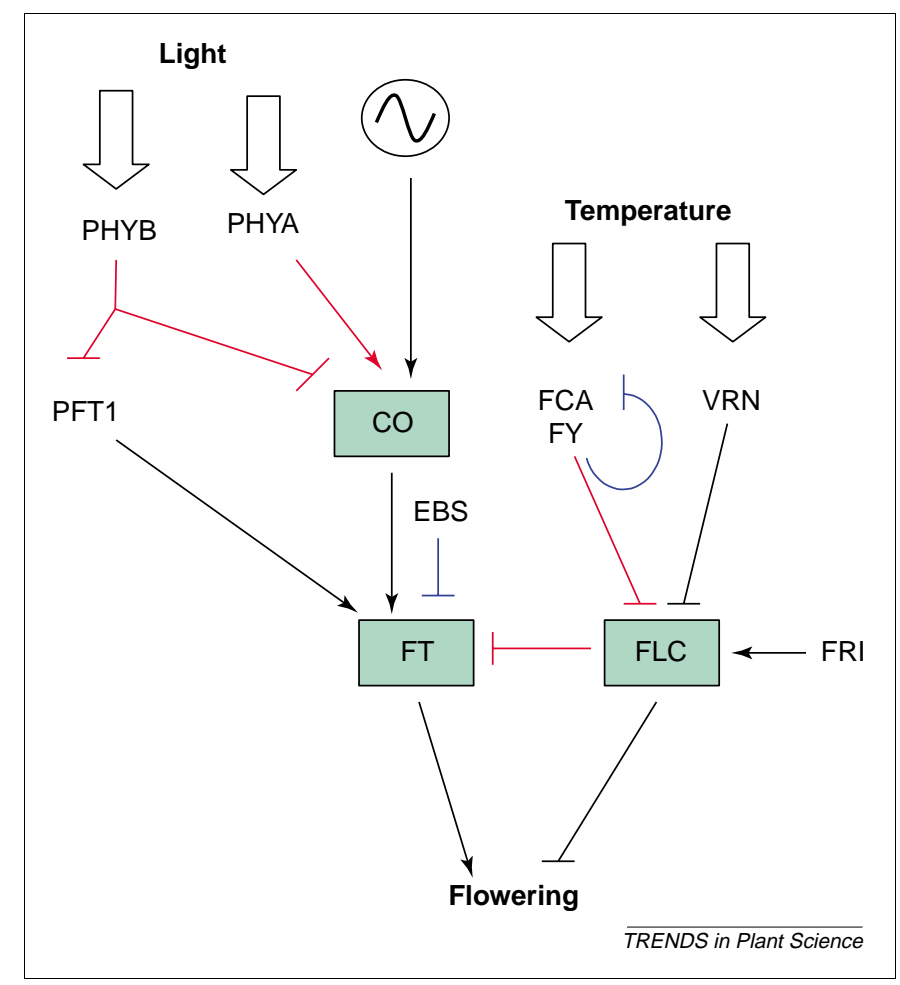

Figure 2. The architecture of signalling webs is the basis for plasticity. Elements shared by different signalling pathways are depicted in green; partially divergent pathways are depicted in red; negative regulation antagonized by the environment is depicted in blue. The wave symbol indicates circadian control.

gene is another repressor of flowering in Arabidopsis but its expression is less sensitive to vernalization than that of FLC. MAF2 could prevent premature fulfilment of the vernalization requirement by brief cold spells. The vernalization response might have evolved through recruitment of a different set of MADS box transcription factors in cereals [7].

In addition to the promoting effect of low temperatures via the vernalization pathway, and the relatively unspecific action mediated by temperature effects on the kinetics of biochemical reactions, a third pathway by which temperature influences flowering is becoming evident from the observation that exposure to low temperatures throughout the growing period delays flowering more than the rate of leaf production in Arabidopsis [8]. In contrast to wild-type strains and mutants of the gibberellin or photoperiod pathways, the fca and fve mutants flower with the same number of leaves irrespective of growth temperature at $16{ }^{\circ} \mathrm{C}$ or $23{ }^{\circ} \mathrm{C}$ [9]. The $F L C$ gene that plays a key role in the vernalization response is not involved in this thermosensory pathway [9].

Light also has more than one action on flowering. The phytochrome and flowering time 1 (pft1, PTF1, GenBank Accession number AY170377) mutant shows delayed flowering, suppresses the early-flowering phenotype of the phytochrome $B$ ( $p h y B, P H Y B$, GenBank Accession number AY466496) mutant and reduces the response to low red to far-red ratios without affecting the response to photoperiod [10]. Thus, these two light signals appear to operate via partially divergent pathways (Figure 2). 


\section{De-etiolating seedlings}

The case of a seedling emerging from the soil provides a clear example of a multiplicity of interacting pathways initiated by a single environmental signal (the transition between full darkness and daily light cycles) that activates phytochromes, cryptochromes and phototropins. Most of the members of these families, including the recently characterized phytochrome $\mathrm{C}$ (phyC, Accession number $\mathrm{X} 17343)[11,12]$, participate in the de-etiolation response. In addition to the direct action of light via these classical photoreceptors, a more indirect action of light can take place via changes in photoassimilate levels. Leaf growth responses to irradiance appear to be partially mediated by the signalling ability of HEXOKINASE 1 (Accession number U28214), which does not depend on its glucose phosphorylation capacity [13].

In the signalling networks that connect environmental cues to growth and development, a complex web of pathways is observed irrespective of the level of resolution. If we scale down from multiple receptors of the light signal to one, we can still distinguish several branches. For example, in the case of phytochrome A (phyA, X17341), different domains of the molecule participate in the control of pathways that can be dissected genetically by mutations in downstream components: molecularly by the relevant regions of target gene promoters and functionally by the strength of the light signal required to activate the pathway $[14,15]$. Furthermore, if we rotate the plane of analysis, phyA is known to control transcription via direct interaction with DNA-binding proteins [16] but it is also able to regulate the abundance of transcription factors involved in photomorphogenesis by reducing CONSTITUTIVE PHOTOMORPHOGENESIS 1 (COP1, Accession number L24437)-mediated targeting to proteasome degradation [17].

\section{Elements shared by different pathways Photomorphogenic signals}

Seedling de-etiolation involves synergic and antagonistic interactions among photoreceptors. Strikingly, some of these interactions can occur even in the absence of a light signal that is necessary to activate one of the participating photoreceptors. For instance, the allele of the blue-light photoreceptor CRYPTOCHROME 2 (CRY2) gene (Accession number U43397) found in the Arabidopsis accession collected from Cape Verde islands enhances the phyAmediated cotyledon-unfolding response to far-red light, which is not directly detected by CRY2 [18]. The mechanisms involved in these interactions are largely unknown, but one exception is the synergism between phyB and phyC, which can be accounted for by the positive effect of phyB on phyC protein levels [11,12].

There are signalling elements shared by several pathways, as revealed by mutations that affect signalling by more than one photoreceptor. Interesting examples are provided by the putative bHLH transcription factor LONG HYPOCOTYL IN FAR-RED 1 (HFR1, Accession number AF324245) and the $\mathrm{Ca}^{2+}$-binding protein SHORT UNDER BLUE LIGHT 1 (SUB1, Accession number AL161512), which are required for normal phyA and cry1 (Accession number NM116961) responses [19,20]. However, these shared players are not necessarily involved in the interactions among photoreceptors [20].

Several mutants uncovered by a photomorphogenic phenotype were later shown to have defects in circadian rhythms and vice versa. The sensitivity to red light reduced 1 (srr1) mutant (SRR1, GenBank Accession number NM125348) shows a de-etiolation phenotype that is weaker and a leaf rhythm phenotype that is stronger than that of the phyB mutant [21]. One possibility is that SRR1 is involved in two different but connected molecular functions, one in light signalling and the other in circadian rhythms. This appears to be the case for cryptochromes in mammals where they are involved in light signalling as well as acting as central components of the circadian clock [22]. Another example is provided by the TIMING OF CAB EXPRESSION 1 (TOC1) gene (Accession number AF272039). The toc1-1 mutant shows defects in circadian rhythms that are consistent with a role in the central oscillator but it retains normal inhibition of hypocotyl growth by light. The toc1-2 allele, which is a null, affects both the circadian rhythm and the hypocotyl response to low light [23]. The PSEUDO-RESPONSE REGULATOR 7 (Accession number NM120359) has been proposed as an early signalling element downstream of phyA that has been recruited to function as an integral component of the circadian oscillator [24]. This intimate connection between light signalling and circadian rhythms appears essential for plasticity because light is a key signal for clock entrainment and the rhythms of sensitivity are crucial for light perception [25-27]. Gating by the clock might help to avoid responses to biologically insignificant fluctuations in the light environment.

The astray mutant of Lotus japonicus shows enhanced nodulation in response to rhizobia and fails to respond normally to light and gravity signals [28]. astray is deficient in a basic leucine zipper, the LONG HYPOCOTYL 5 (HY5, Accession number AB005456) homologue of Lotus japonicus [28]. Clearly, the same gene can play significant roles in the response to seemingly unrelated environmental cues.

\section{Integration of pathways controlling flowering}

Recent advances in the understanding of the mechanisms involved in the control of flowering by environmental cues reveal shared and specific players. In Arabidopsis plants grown under long days, the presence of light perceived by photoreceptors such as cry2 and phyA coincides with high mRNA levels of the CONSTANS (CO) gene (Accession number X94937), whose expression is under circadian control [26,27]. Light is required for the stabilization of $\mathrm{CO}$, which in darkness undergoes proteasome-mediated degradation [29]. As a result of this coincidence, the expression of the FLOWERING LOCUS T $(F T)$ gene (Accession number AB027504) is dramatically increased and this leads to the promotion of flowering. Under short days, the waveform of $C O$ expression is only slightly affected, but the main difference is that by the time $C O$ expression is high, light is no longer present and the expression of $F T$ remains low [26,27]. In short-day plants such as rice, regulation of the $F T$ orthologue (Accession number BAB61027) by the $\mathrm{CO}$ orthologue (AB041839) is 
reversed, resulting in the suppression of flowering under long days [30]. FT appears to be a key integrator of different floral induction signals because the effects of the phyB mutation [10] [31], ambient temperature [9], and reduced FLC expression [32] also involve enhanced expression of FT. Similarly, SUPPRESSOR OF OVEREXPRESSION OF CO 1 (SOC1, Accession number AY007726) [32,33] and AGAMOUS-LIKE 24 (Accession number AF005158) [34,35] are MADS box transcription factor-encoding genes that also integrate endogenous and environmental (e.g. vernalization) cues. Thus, signalling appears to converge to several parallel transcription factors and not to concentrate on a single player. The photoperiodic and low red to far-red signals converge upstream FT by stabilizing CO [29]. The shape of the network emerges as a consequence of multiple points of parallel and sequential signalling convergence.

\section{Environmental signals antagonize negative regulators}

The external signal often appears to influence the balance between positive and negative regulators, thereby allowing the positive regulators to promote the response. For example, COP1, DE-ETIOLATED 1 (DET1, Accession number L33695) [36,37], and COP9 (Accession number L33695) [38] are nuclear proteins that repress photomorphogenesis in darkness and have animal counterparts. COP1 promotes ubiquitin-mediated proteolysis of a subset of positive regulators, including HY5 [17] and LONG AFTER FAR-RED (LAF1, Accession number Z95744) [39]. Light promotes translocation of COP1 to the cytoplasm allowing de-etiolation, partly through the action of HY5 and LAF1. Similarly, in cop1 mutants, HY5 and LAF1 accumulate in the dark and photomorphogenesis proceeds in the absence of light.

DET1 binds in vitro to nonacetylated $\mathrm{N}$-terminal tails of the core histone $\mathrm{H} 2 \mathrm{~B}$ in the context of the nucleosome and interacts with DAMAGED DNA BINDING PROTEIN 1 (DDB1, Accession number NP_192451) [36,37]. The DET1-DDB1 complex could interact with chromatin via association with the nonacetylated H2B tail, negatively regulating transcription. Light could recruit histone acetyltransferase via interaction with DDB1, acetylate histone, release the DET1 complex and therefore allow the expression of photomorphogenic genes [36,37]. DDB1 has recently been reported in complexes containing the COP9 signalosome in fibroblasts [40]. The retinoblastomarelated E2F transcription factor is degraded by the ubiquitin-proteasome pathway in response to light, suggesting that this regulation is necessary to activate gene expression required for photomorphogenesis [41].

Vernalization down regulates the expression of $F L C$ and maintaining this repression requires epigenetic regulation mediated by VERNALIZATION 2 (Accession number AF284500), a gene with similarity to the polycomb group of proteins [42]. The levels of FLC are crucial for the vernalization response and the interaction between FCA (Accession number Z82992) and FY (Accession number NM_121351) appears to regulate FLC mRNA $3^{\prime}$ end formation providing a fine-tuning mechanism [43].

Mutations at EARLY BOLTING IN SHORT DAYS (EBS, Accession number N96619) or TERMINAL
FLOWER 2 (TFL2, Accession number NM121775) accelerate flowering by specifically de-repressing FT [44,45]. EBS and TFL2 contain domains that are normally involved in chromatin remodelling. Thus, the response to environmental signals such as daylength requires $F T$ expression to be repressed by EBS and TFL2 to give room for up-regulation of $F T$ by these signals.

Rapid responses to the environment can require rapid shifts in the transcriptome. Changes in mRNA levels of hormone-related genes accompany early cryptochrome 1-mediated inhibition of hypocotyl growth in response to blue light [46]. Although transcription is a key target in the regulation of changes in gene expression underlying developmental responses, mRNA degradation is involved in the control of the steady-state mRNA levels and an estimated 1\% of Arabidopsis genes correspond to unstable messages [47]. The analysis of the behaviour of these genes in a different set of expression experiments in Arabidopsis indicates a potential role in responses to mechanical simulation and other signals known to induce rapid responses [47].

\section{Regulatory loops \\ Environmental cues activate signalling and negative regulation of signalling}

There are several examples where an environmental cue initiates downstream signalling and sets into motion mechanisms that negatively regulate that signalling. The RGL2 gene (Accession number NM111216) negatively regulates seed germination responses to gibberellin [48]. The expression of $R G L 2$ is up-regulated by seed imbibition, which is a pre-requisite for germination. Light causes COP1 migration to the cytosol but it also enhances the expression of SPA1 (Accession number AF135455) and the residual COP1 in the nucleus is activated by SPA1, which enhances its E3 activity reducing the photomorphogenic response [39]. Furthermore, the active form of phytochrome migrates to the nucleus where it interacts and presumably activates PHYTOCHROME INTERACTING FACTOR 3 (Accession number AF100166) [49], a negative regulator of phytochrome signalling [50].

\section{Mutually regulated regulators}

PHYTOCHROME KINASE SUBSTRATE 1 (PKS1, Accession number AF149053) is phosphorylated by phyA [51]. The expression of PKS1 and of PKS2 (Accession number NM101294, the closest homologue of PKS1 in the Arabidopsis genome) is rapidly and transiently enhanced by light perceived by phyA [52]. Both $p k s 1$ and $p k s 2$ show enhanced responses to pulses of far-red light perceived by phyA. However, the pks1 pks2 double mutant shows a wild-type behaviour [52]. Furthermore, transgenic seedlings overexpressing PKS1 or PKS2 show the same phenotype as the knockout mutants. PKS1 and PKS2 interact physically, and PKS1 levels are negatively regulated by PKS2 under the physiological conditions where the phenotype is observed [52]. These observations are consistent with a model where PKS1 and PKS2 are positive regulators of phyA signalling and mutual negative regulators. In agreement with this view, the effect of enhanced PKS1 levels is larger in the pks 2 background 
(i.e. in the absence of the negative regulation imposed by PKS2) [52]. These loops are therefore a source of homeostasis.

\section{Future directions}

Previous sections illustrate the recent advances in the search for players and the characterization of their molecular function in the signalling networks controlling plant plasticity. One of the future challenges is to elucidate the function of these players in the network as a whole because complex systems have emergent properties that are not entirely obvious when the elements are considered in isolation.

The mechanisms that involve an active repressor in the absence of a signal could result from an evolutionary sequence where the repressor of developmental progression is required to establish the need for a signal and thus achieve coordination with the environment. However, mutant analysis shows that negative regulators not only prevent the 'response' in the absence of the signal but also ensure that in the presence of the signal an exaggerated response does not occur. As noted above, some of the negative regulation is enhanced by the signal itself as part of a feedback loop. This post signal function can be understood intuitively by arguing, for instance, that termination of signalling is required to reset the system for a new signal. Temporal and spatial restrictions are obvious functions of negative regulation but not the only ones [53]. Mathematical modelling and experiments with engineered gene circuits indicate that a network with negative feedback is more stable than an unregulated network [54]. Noise in cellular processes appears to arise mainly during translation because a single mRNA transcript can produce multiple copies of a protein [55]. The principle of negative feedbacks is to respond to deviations from the normal parameters providing a simple mechanism to attenuate noise [55]. Complex networks involving multiple feedback loops provide robustness [55].

The occurrence of downstream signalling elements shared by different receptors could be the consequence of repeated use of a limited number of elements during the course of evolution or a means to coordinate different cues. In the first case, cross talk should be avoided by temporal and/or spatial discrimination of usage of the element by different signals. In the second case, cross talk is the goal but the occurrence of cross-talk in mammalian cells has been challenged [56]. Plant plasticity provides a fertile area where these ideas can be tested.

\section{Acknowledgements}

We are grateful to the Juan March foundation (Madrid, Spain) for providing the forum where we initiated the discussion of the ideas presented here (Plasticity in Plant Morphogenesis, 24-26 February 2003).

\section{References}

1 Jürgens, G. (2001) Apical-basal pattern formation in Arabidopsis embryogenesis. EMBO J. 20, 3609-3616

2 Queitsch, C. et al. (2002) Hsp90 as a capacitor of phenotypic variation. Nature 417, 618-624

3 Bergman, A. and Siegal, M.L. (2003) Evolutionary capacitance as a general feature of complex gene networks. Nature 424, 549-552
4 Pinyopich, A. et al. (2003) Assessing the redundancy of MADS-box genes during carpel and ovule development. Nature 424, 85-88

5 Johanson, U. et al. (2000) Molecular analysis of FRIGIDA, a major determinant of natural variation in Arabidopsis flowering time. Science 290, 344-347

6 Michaels, S.D. et al. (2003) Attenuation of FLOWERING LOCUS C activity as a mechanism for the evolution of summer-annual flowering behavior in Arabidopsis. Proc. Natl. Acad. Sci. U. S. A. 100, 10102-10107

7 Trevaskis, B. et al. (2003) MADS box genes control vernalizationinduced flowering in cereals. Proc. Natl. Acad. Sci. U. S. A. 100, 13099-13104

8 Mazzella, M.A. et al. (2000) Temperature-dependent internode elongation in vegetative plants of Arabidopsis thaliana lacking phytochrome B and cryptochrome 1. Planta 210, 497-501

9 Blázquez, M.A. et al. (2003) A thermosensory pathway controlling flowering time in Arabidopsis thaliana. Nat. Genet. 33, 168-171

10 Cerdán, P. and Chory, J. (2003) Regulation of flowering time by light quality. Nature 423, 881-885

11 Monte, E. et al. (2003) Isolation and characterization of phyC mutants in Arabidopsis reveals complex crosstalk between phytochrome signaling pathways. Plant Cell 15, 1962-1980

12 Franklin, K.A. et al. (2003) Mutant analyses define roles for phytochrome C in Arabidopsis photomorphogenesis. Plant Cell 15, 1981-1989

13 Moore, B. et al. (2003) Role of Arabidopsis glucose sensor HXK1 in nutrient, light, and hormonal signaling. Science 300, 332-336

14 Casal, J.J. et al. (2002) The serine-rich N-terminal domain of oat phytochrome A helps regulate light responses and subnuclear localization of the photoreceptor. Plant Physiol. 129, 1127-1137

15 Yanovsky, M.J. et al. (2002) Missense mutation in the PAS2 domain of phytochrome A impairs subnuclear localization and a subset of responses. Plant Cell 14, 1591-1603

16 Quail, P.H. (2002) Phytochrome photosensory signalling networks. Nat. Rev. Mol. Cell Biol. 3, 85-93

17 Osterlund, M.T. et al. (2000) Targeted destabilization of HY5 during light-regulated development of Arabidopsis. Nature 405, 462-466

18 Botto, J.F. et al. (2003) The Cape Verde Islands allele of cryptochrome 2 enhances cotyledon unfolding in the absence of blue light in Arabidopsis. Plant Physiol. 133, 1547-1556

19 Guo, H. et al. (2001) SUB1, an Arabidopsis $\mathrm{Ca}^{2+}$-binding protein involved in cryptochrome and phytochrome coaction. Science 291, $487-490$

20 Duek, P.D. and Fankhauser, C. (2003) HFR1, a putative bHLH transcription factor, mediates both phytochrome A and cryptochrome signalling. Plant J. 34, 827-836

21 Staiger, D. et al. (2003) The Arabidopsis SRR1 gene mediates phyB signaling and is required for normal circadian clock function. Genes Dev. 17, 256-268

22 Selby, C.P. et al. (2000) Functional redundancy of cryptochromes and classical photoreceptors for nonvisual ocular photoreception in mice. Proc. Natl. Acad. Sci. U. S. A. 97, 14697-14702

23 Más, P. et al. (2003) Dual role of TOC1 in the control of circadian and photomorphogenic responses in Arabidopsis. Plant Cell 15, 223-236

24 Kaczorowski, K.A. and Quail, P.H. (2003) Arabidopsis PSEUDORESPONSE REGULATOR7 (PRR7) is a signaling intermediate in phytochrome-regulated seedling deetiolation and phasing of the circadian clock. Plant Cell 15, 2654-2665

25 Anderson, S.L. et al. (1997) Attenuation of phytochrome A and B signaling pathways by the Arabidopsis circadian clock. Plant Cell 9, $1727-1743$

26 Suárez-López, P. et al. (2001) CONSTANS mediates between the circadian clock and the control of flowering in Arabidopsis. Nature 410, 1116-1120

27 Yanovsky, M.J. and Kay, S.A. (2002) Molecular basis of seasonal time measurement in Arabidopsis. Nature 419, 308-312

28 Nishimura, R. et al. (2002) A Lotus basic leucine zipper protein with a RING-finger motif negatively regulates the developmental program of modulation. Proc. Natl. Acad. Sci. U. S. A. 99, 15206-15210

29 Valverde, F. et al. (2004) Photoreceptor regulation of CONSTANS protein in photoperiodic flowering. Science 303, 1003-1006

30 Hayama, R. et al. (2003) Adaptation of photoperiodic control pathways produces short-day flowering in rice. Nature 422, 719-722 
31 Halliday, K.J. et al. (2003) Phytochrome control of flowering is temperature sensitive and correlates with expression of the floral integrator FT. Plant J. 33, 875-885

32 Hepworth, S.R. et al. (2002) Antagonistic regulation of flowering-time gene SOC1 by CONSTANS and FLC via separate promoter motifs. EMBO J. 21, 4327-4337

33 Moon, J. et al. (2003) The SOC1 MADS-box gene integrates vernalization and gibberellin signals for flowering in Arabidopsis. Plant J. $35,613-623$

34 Michaels, S.D. et al. (2003) AGL24 acts as a promoter of flowering in Arabidopsis and is positively regulated by vernalization. Plant J. 33, 867-874

$35 \mathrm{Yu}$, H. et al. (2002) AGAMOUS-LIKE 24, a dosage-dependent mediator of the flowering signals. Proc. Natl. Acad. Sci. U. S. A. 99, 1633616341

36 Benvenuto, G. et al. (2002) The photomorphogenesis regulator DET1 binds the amino-terminal tail of histone H2B in a nucleosome context. Curr. Biol. 12, 1529-1534

37 Schroeder, D. et al. (2002) De-Etiolated 1 and Damaged DNA Binding Protein 1 interact to regulate Arabidopsis photomorphogenesis. Curr. Biol. 12, 1462-1472

38 Wei, N. and Deng, X.W. (2003) The COP9 signalosome. Annu. Rev. Cell Dev. Biol. 19, 261-286

39 Seo, H.S. et al. (2003) LAF1 ubiquitination by COP1 controls photomorphogenesis and is stimulated by SPA1. Nature 424, 995-999

40 Groisman, R. et al. (2003) The ubiquitin ligase activity in the DDB2 and CSA complexes is differentially regulated by the COP9 signalosome in response to DNA damage. Cell 113, 357-367

41 del Pozo, J.C. et al. (2002) Arabidopsis E2Fc functions in cell division and is degraded by ubiquitin-SCF ${ }^{\mathrm{AtSKP} 2}$ pathway in response to light. Plant Cell 14, 3057-3071

42 Gendall, A.R. et al. (2001) The VERNALIZATION 2 gene mediates the epigenetic regulation of vernalization in Arabidopsis. Cell 107, $525-535$

43 Simpson, G.G. et al. (2003) FY is an RNA $3^{\prime}$ end-processing factor that interacts with FCA to control the Arabidopsis floral transition. Cell $113,777-787$
44 Piñeiro, M et al. (2003) EARLY BOLTING IN SHORT DAYS is related to chromatin remodeling factors and regulates flowering in Arabidopsis by repressing FT. Plant Cell 15, 1552-1562

45 Takada, S. and Goto, K. (2003) TERMINAL FLOWER 2, an Arabidopsis homolog of HETEROCHROMATIN PROTEIN 1, counteracts the activation of FLOWERING LOCUS T by CONSTANS in the vascular tissues of leaves to regulate flowering time. Plant Cell 15 2856-2865

46 Folta, K.M. et al. (2003) Genomic and physiological studies of early cryptochrome 1 action demonstrate roles for auxin and gibberellin in the control of hypocotyl growth by blue light. Plant J. 36, 203-214

47 Gutiérrez, R.A. et al. (2002) Identification of unstable transcripts in Arabidopsis by cDNA microarray analysis: rapid decay is associated with a group of touch- and specific clock-controlled genes. Proc. Natl. Acad. Sci. U. S. A. 99, 11513-11518

48 Lee, S. et al. (2002) Gibberellin regulates Arabidopsis seed germination via $R G L 2$, a GAI/RGAI-like gene whose expression is up-regulated following imbibition. Genes Dev. 16, 646-658

49 Martínez-García, J.F. et al. (2000) Direct targeting of light signals to a promoter element-bound transcription factor. Science 288, 859-863

$50 \mathrm{Kim}$, J. et al. (2003) Functional characterization of Phytochrome Interacting Factor 3 in phytochrome-mediated light signal transduction. Plant Cell 15, 2399-2407

51 Fankhauser, C. et al. (1999) PKS1, a substrate phosphorylated by phytochrome that modulates light signaling in Arabidopsis. Science 284, 1539-1541

52 Lariguet, P. et al. (2003) A growth regulatory loop that provides homeostasis to phytochrome A signaling. Plant Cell 15, 2966-2978

53 Freeman, M. (2000) Feedback control of intercellular signalling in development. Nature 408, 313-319

54 Hasty, J. et al. (2002) Engineered gene circuits. Nature 420, 224-230

55 Rao, C.V. et al. (2002) Control, exploitation and tolerance of intracellular noise. Nature 420, 231-236

56 Noselli, S. and Perrimon, N. (2000) Are there close encounters between signaling pathways? Science 290, 68-69

\section{Letters to Trends in Plant Science}

If you wish to comment on an article recently published in Trends in Plant Science or would like to discuss issues of general current interest to plant scientists, please write a Letter to the Editor. Letters should be no more than 750 words long with a maximum of 12 references and one small figure. Letters should be e-mailed to plants@current-trends.com.

The decision to publish rests with the Editor, and the author(s) of any Trends in Plant Science article criticized in a Letter will normally be invited to reply. 